\title{
Abstracts of Spinal Injury Association Meeting, 1986
}

\author{
Held in San Francisco, California, U.S.A. March 1986 \\ These were kindly collected and abstracted by Dr David F. Apple, fr., M.D.
}

\author{
The Frankel grade versus the motor index score \\ David F. Apple, Jr., M.D., Carolyn Albright, R.N., Jane Huckeba, L.P.N., James A. \\ Murphy, B.M.E., Bob Pollard, M.A. \\ Shepherd Spinal Center, 2020 Peachtree Road, N.W., Atlanta, Georgia 30309, U.S.A.
}

The authors reviewed the medical records of 501 traumatically injured patients with spinal cord injury. Motor index scores as well as the Frankel Grade at discharge were tabulated. Patients were divided into five study groups. Group I was $\mathrm{Cl}$ to $\mathrm{C} 4$, Group II was $\mathrm{C} 5$ to $\mathrm{C} 8$, Group III was T1 to T11, Group IV was T12 to L1, and Group V was L2 to the sacrum. There were sufficient numbers in all Frankel Grades except $\mathrm{E}$ to make statistical analysis possible. There was good correlation between injury level and combined motor index score for Frankel Grade A, B, and C. There was no correlation possible for Frankel Grade D due to the random combined motor index score for that grade. As a result of the analysis, the authors suggested utilising injury level in combination with the combined motor index score to give a better indication of the function of the patient than the Frankel Grade alone, especially in the Frankel D Group. When sensation is present below the level of injury, a plus $(+)$ would be used to indicate this. Thus a C6 central cord syndrome would thus be labelled: C6 - 30+. Utilisation of this system would give all the information that the Frankel specification gives with the added dimension of indicating precisely the degree of function.

\section{Relationships between near surface blood flow and altered sensations among spinal cord injured veterans \\ Richard A. Sherman, Ph.D., Jeffrey L. Ernst, Ph.D., Janusz Markowski, M.D. \\ Department of Clinical Investigation, D. D. Eisenhower Army Medical Center, Fort Gordon, Georgia 30905, U.S.A.}

Thirty-nine patients with clinical diagnoses of complete transverse spinal cord destruction were interviewed concerning any sensations that they felt below the level at which normal feelings were evident. All reported experiencing various feelings most of the time and 38 reported that some of these sensations were usually quite painful. Videothermographs showing differences in skin temperature of 0.1 degrees celsius were taken to evaluate blood flow patterns to a depth of $1.5 \mathrm{~cm}$. Changes in blood flow patterns were found to correlate highly with the level at which sensations changed from normal to abnormal and correlate virtually exactly with the locations of pain reported from supposedly insensitive areas. In every case, near surface temperature decreased by at least $2^{\circ}$ celsius below the demarcation line between normal and abnormal sensations. In all but one case, painful areas below the level of normal sensation were 1 to $5^{\circ}$ warmer than the surrounding tissue. The areas delineated by the temperature differences did not correspond to dermatomes or thermatomes. 


\section{Head trauma in the spinal-injured patient}

Julian E. Bailes, M.D., Matthew R. Quigley, M.D., Vernon Keenan, III, Leonard J.

Cerullo, M.D., Paul R. Meyer, M.D.

Division of Neurosurgery, Northwestern University Medical School, 303 East Chicago Avenue, Chicago, Illinois 60611, U.S.A.

The head and neck should be considered together when evaluating the patient in the initial care setting. Not only is primary head injury possible but there may also be cerebral injury secondary to involvement of the carotid or vertebral arteries in the neck. Between 1972 and 1985, 2300 patients were admitted to the Midwest Regional Spinal Cord Injury Care System, 364 having associated head injuries. Fifty-eight per cent of all head injured patients also had fractures in the cervical spine. Fifteen per cent had fractures of the thoracic spine, $13 \%$ had fractures at the thoracic lumbar junction and $3 \%$ had fractures of the lumbar spine. Eleven per cent had no skeletal injury. Nineteen per cent of the patients had no neurological injury. Fortytwo per cent had an incomplete neurological injury and 39\% of the patients reviewed had a complete neurological injury. Forty-six of the 364 head injury patients had multiple trauma in addition to head and spine injuries. Of the head injuries, twelve patients (or 3\%) had scalp lacerations., $16(4 \%)$ had skull fractures, and $12(3 \%)$ had mandible fractures. The authors concluded that a cognicance must be held for the possibility of coexisting head and spinal injury. When significant head impact has occurred, or there is evidence of a compression of the cerebral circulation, the radiological evaluation should include computerised tomography of the head and possibly cervical and cerebral angiography. As soon as the patient has had spinal immobilisation, prompt neurosurgical evaluation is required for any significant cerebral lesions that may be present.

\section{The co-incidence of spinal cord injury and closed head injury: results of a longitudinal investigation}

J. Scott Richards, PhD., Kris Hagglund, B.A.

Spain Rehabilitation Center, 1717 6th Avenue South, Birmingham, Alabama 35233, U.S.A.

There is a greater awareness of the fact that the absence of neurological indicators (e.g. positive CAT scan, EEG, neurological examination) of closed head injury does not ensure that a closed head injury has not occurred. Increasing sophistication in the field of neuropsychological assessment has demonstrated that many persons with a brief loss of consciousness and negative neurological evaluations often have disabling cognitive and behavioural sequelae from the closed head injury.

A neuropsychological battery study consisting of well-validated tests involving no motor output was developed. Trained examiners administered this battery of tests to all newlyinjured SCI patients within 3 weeks of admission to an acute care spinal injury center, and again approximately 6 months postdischarge. Repeat testing is methodologically critical with this group as their level of cognitive functioning generally tends to be low. Without repeat testing (and hence the chance to observe improvements in scores) it would be difficult to state whether low scores were a function of closed head injury or pre-existing cognitive ability.

The authors evaluated 67 patients with the initial and repeat neuropsychological battery of tests. Preliminary results showed that on some of the tests there was no substantial change across time. However, there was a notable gain on the Halstead-Reitan Category Test, one of the most sensitive indicators of impairment in cognitive function. There was little change in intelligence scores over time, but Wechsler Memory Scale scores improved over time as did 
scores on verbal fluency. Final data analysis is being completed, including ratings by three neuropsychologists as to the presence/absence of $\mathrm{CHI}$ in this group and its potential impact.

\section{Biomechanical and radiological analysis of the cervical transverse ligament (of the atlas vertebra) \\ Thomas R. Highland, M.D., Paul C. Begeman, Ph.D., and Gino G. Salciccioli, M.D. Department of Orthopaedic Surgery, Wayne State University School of Medicine, 540 East Canfield Avenue, Detroit, Michigan 48201, U.S.A.}

The authors isolated $\mathrm{C} 1$ and $\mathrm{C} 2$ of 22 fresh cadaver specimens with the transverse ligament being the only major structure left intact. A Jefferson fracture of $\mathrm{Cl}$ was created by cutting both the anterior and posterior arches. A pin was placed through a predrilled hole in each lateral mass of $\mathrm{Cl}$ and these pins were attached to the Instrom machine to apply tension to the transverse ligament. The specimens consisting of $\mathrm{Cl}$ and transverse cervical ligament were stressed.

The authors concluded the following:

1. The force it took to rupture the transverse ligament was significantly smaller than other studies had shown.

2. On the $\mathrm{AP}$ open mouth view of $\mathrm{Cl}$ and $\mathrm{C} 2$, if the total lateral displacement of $\mathrm{Cl}$ is $7 \mathrm{~mm}$ or more this represents a rupture of the transverse cervical ligament associated with a burst fracture of the atlas.

3. The Atlanto-Dens interval on the radiograph taken after the specimen was pinned and stressed was not always greater than $3 \mathrm{~mm}$ in the unmagnified view.

4. The place of rupture of the ligament was variable.

Based on these findings we felt that the concept of the transverse cervical ligament being the primary restraint of the atlantal axial joint should be further examined. Perhaps other structures are more important than has been previously thought, and further studies require to be done.

\section{Magnetic resonance imaging in the evaluation of spinal cord injured children}

A. J. Gelman. D.O., R. R. Betz, M.D., G. J. DeFilipp, M.D., M. Mesgarzadh, M.D. Shriners Hospital, 8400 Roosevelt Boulevard, Philadelphia, Pennsylvania 19152, U.S.A.

Computerised axial tomography $(\mathrm{CT})$ is now used routinely in the evaluation of spinal cord trauma, but the value of magnetic resonance imaging (MRI) has yet to be established. The purpose of this paper is to compare MRI scanning with CT myelography to determine its indications and usefulness.

\section{Method}

Forty-three patients with subacute or chronic spinal cord injury seen in the Philadelphia Unit of Shriners Hospitals were reviewed. The average age of these patients was 16 years (range: 4 months to 21 years). Studies include MRI scans (43 patients), plain radiographs (43), myelograms (24), metrizamide CT scans (24), tomograms (10), and plain CT scans (8).

Results

MRI scans were superior to CT in evaluating post-traumatic syrinx. Thirteen patients had post-traumatic syrinxes, only two of which were seen on delayed metrizamide CT scans. The 
MRI was superior for establishing the physiological status of the intervertebral disc. It was also effective in evaluating spinal cord physiology. One 13-year-old child with $\mathrm{C} 4$ complete quadriplegia demonstrated an abnormal signal in the spinal cord when all other radiographic evaluation, including metrizamide Ct scan, was normal. Another patient, 4 months of age, showed a widened $\mathrm{C} 2$ syndesmosis but otherwise normal radiographic studies. including CT myelogram. However, there was an abnormal signal on the MRI scan at C5-C6-C7, which corresponded to his clinical level.

Direct comparison of metrizamide CT scanning with MRI scanning revealed that MRI scans were effective in screening for areas of spinal cord compression, but metrizamide CT scan was superior and necessary to evaluate a patient fully before considering surgery. Twentythree MRI scans were obtained in patients having had prior internal spinal fixation. In four of these patients, there was artifact obscuring some data, but the information obtained was still useful, and in two patients the artifact was severe. In the other 17 patients there was no artifact secondary to the metal.

\section{Conclusions}

MRI scanning is indicated in the evaluation of subacute or chronic spinal cord injured patients for determination of post-traumatic syrinx, disc pathology, or physiological status of the spinal cord. It appears to be helpful in screening for the level of injury, spinal column alignment, and extrinsic spinal cord compression. Metrizamide CT scan remains an essential study before considering surgery for spinal cord decompression. The presence of internal vertebral column fixation is not a contraindication to MRI scanning and, in the majority of cases, permits an accurate study.

\section{Continuous versus intermittent turning: analysis of direct nursing time and treatment of acute spinal cord injury \\ J. M. Bugaresti, M.D., C. H. Trator, M.D., Ph.D., J. P. Szalai, Ph.D., H. Chin-Sang, M.D., R. Magissano, M.D. \\ Sunnybrook Medical Center, University of Toronto, 399 Bathurst Street, Toronto, Ontario M5T 2S8, Canada}

The authors did a controlled study to compare direct nursing care time for the continuous and intermittent care method. Fifty-two patients were entered into the study. The authors concluded that the study failed to demonstrate specifically a significant difference between the use of an automated continuous side-to-side turning bed compared with manual intermittent turning with regard to the reduction of complications and the length of stay in an acute hospital. Continuous turning appeared to be helpful for the management of patients in traction, and for patients at risk for respiratory complications.

\section{Posterior cervical fusion under local anesthesia (the awake patient is the ultimate spinal cord monitor)}

Jack Zigler, M.D., Neil Rockowitz, M.D., Daniel Capen, M.D., Russell Nelson, M.D., Robert Waters, M.D.

Rancho Los Amigos Medical Center, University of Southern California, Downey, California 90242, U.S.A.

The authors reviewed twenty-eight consecutive patients operated on in the Rancho Los Amigos Medical Center where posterior cervical wiring and fusion with iliac bone graft was 
done under local anaesthesia. Using this technique, care should be taken not to expose any levels which are not to be included in the level of fusion. Communication between the surgeon and patient is important during patient positioning, dissection, and passage of internal fixation wires. The bone graft can be taken under local anaesthesia. Once the cervical spine is fixed with wires, additional sedation may be used if the patient is uncomfortable. In patients with high neurological level lesions, the bone graft site is often anaesthetic.

The authors did not decorticate the posterior elements, laying the cancellous bone over the exposed lamina and facet joints. There were no significant intra-operative complications and no major neurological complications. The authors concluded that patients with an unstable cervical spine and variable degrees of neurological injury can be managed in a teaching center by posterior cervical stabilisation and fusion under local anaesthesia. The advantage of local anaesthesia allows patient position and interaction during crucial moments of spinal manipulation. The technique is generally well tolerated by patients and no untoward complications occurred as a result of using this technique.

\section{The mechanical principle of external skeletal fixation in dorsal stabilisation of the thoracic and lumbar spine}

Patrick Kluger, M.D. and Frederik H. Schmidt, M.D.

Werner-Kluger-Klinik, Department of Spinal Cord Injuries, 3590 Bad-Wildungen-West, Federal Republic of Germany

The authors discussed the use of MAGERL's fixateur externe, the management instrument for spinal cord injury, specifically used in cases where there was infection, open injuries and generally poor condition. The authors desire to develop instruments that would provide the mechanical characteristics of external fixation to be implanted directly into the spine. They elected to try the DICK instruments and evaluated them in 45 cases between 1983 and 1985 . Difficulties encountered with the threaded rods and with the Schanz screws prompted the development of new instrumentation. Instrumentation was used in 29 cases with the patients being mobilised a week after surgery provided with a brace when out of bed for 3 to 4 months. The authors reported that there was no deterioration of neurological function, no implant fracture or loosening, and concluded that it was a good procedure.

\section{Management of non-contiguous vertebral fractures \\ David S. Tearse, M.D., James S. Keene, M.D., Denis S. Drummond, M.D. \\ University of Wisconsin, Spinal Injuries Center, Madison, Wisconsin, U.S.A.}

Griffin found a 3.2\% incidence of non-contiguous injuries in 155 thoracolumbar fractures. Calenoff found a $4.5 \%$ incidence of non-contiguous vertebral fractures in a large series of patients with spinal cord injuries. He noted that the second and third level of bony injury is not often diagnosed. The management and results of treatment of these non-contiguous fractures is addressed in this study.

The authors reviewed 78 consecutive patients with acute thoracolumbar fractures. The major level of injury was defined as that level which appeared most unstable on standard radiographs and CT scans, or was associated with a neurological deficit. The type of injury, angle of deformity and percentage displacement were determined at all levels of injury. Thirteen of 78 patients had non-contiguous fractures and three had multiple fractures. Thus, 
they concluded that non-contiguous vertebral fractures are common. Therefore, patients with fractures at one level should have a radiological examination of the entire spine. If noncontiguous fractures are evident on the standard radiograph, all levels of injury should be thoroughly evaluated with computerised tomography. If the minor level is unstable or there is doubt concerning its stability, it should be included within the instrumentation. This method of treatment may require instrumentation of very long segments of spine and severely restrict motion. However, short segmental fusion which only encompasses the unstable segment, and removal of the rods after the fusions are solid may prevent deformities and enhance subsequent mobility of the spine.

The efficacy of surgical management for scoliosis in myelomeningocele: correction of deformity and alteration of functional status

John M. Mazur, M.D., D. R. V. Dickens, M.D., W. G. Doig, M.D., M. B. Menelaus, M.D. Southern Illinois University School of Medicine, Department of Surgery, P.O. Box 3926, Springfield, Illinois 62708, U.S.A.

Forty-nine myelomeningocele patients who had undergone surgery for scoliosis were studied to determine the extent of correction and the effect on function. Fifteen patients underwent posterior fusion with Harrington distraction rods; seven patients underwent anterior Dwyer instrumentation and fusion; and twenty-seven patients underwent combined anterior and posterior spine fusion with Harrington and Dwyer instrumentation.

Posterior fusion with Harrington distraction rods extending to the sacrum combined with anterior fusion with Dwyer instrumentation gave the best results as assessed by fusion rate, improvement in sitting balance and correction of scoliosis, pelvic obliquity, lumbar lordosis and torso decompensation. Improvement in overall function occurred in only one half of the patients despite correction of the spinal deformity. The ability to walk or to be mobile in a wheelchair was adversely affected by spinal fusion. A normal lumbar lordosis must be maintained in order to minimise the risk of sacral and ischial pressure sores. There was a high rate of deep wound infections and neurological complications following posterior spinal fusion. None following anterior fusion. Finally, it is stated that if at all possible, spinal fusion should be avoided in high level spina bifida patients who are able to walk.

\section{Arm positioning for the spinal cord injured patient}

Kim Chapman, O.T.R., B.H.S. and Jill Uliano, O.T.R., B.H.S.

The Lucerne Spinal Injury Center, 818 South Main Lane, Orlando, Florida 32801, U.S.A.

Of 117 patients surveyed at the Lucerne Spinal Injury Center, 66 (54\%) complained of shoulder pain during their initial admission. In order to correct this, three basic positions were used in positioning the arm in the quadriplegic and were incorporated into a regular turning and positioning program. The most important feature of arm positioning in the side lying posture was the use of an axillary chest pillow placed perpendicular under the patient's trunk. This was coupled with a head pillow of corresponding height to maintain proper spinal 
alignment. This positioning serves to bridge the glenohumeral joint off the mattress. The lower arm was placed in $90^{\circ}$ of abduction and $90^{\circ}$ of external rotation with either elbow flexion or extension, and placed on an arm board with foam pad. The upper arm was padded and placed in slight extension.

With the patient in the supine position, the arms were placed in $90^{\circ}$ of abduction, full external rotation and full elbow extension. The upper extremities were placed on the armboards and strapped down in order to maintain their position. The third position involved placing the upper extremities in elbow flexion, external rotation and as much abduction as the patient can tolerate. Arm positions and equipment were developed for use on a Roto Rest bed.

Comparative findings in 15 patients revealed that those patients placed on the arm positioning program had a greater percentage charge in increased shoulder range during their admission than those who were not placed on the program during their stay.

The author's concluded that the modified arm positioning program was an effective method of assisting in the alleviation of shoulder pain and soft tissue contractures in the quadriplegic patient.

\section{Urodynamic evaluation in spinal cord injury patients: how much is needed? L. Keith Lloyd, M.D. and Keith V. Kuhlemeier, Ph.D. University of Alabama Medical Center, Birmingham, Alabama, U.S.A.}

There is currently no general agreement on how urodynamic testing should be performed in spinal cord injured patients. This study was designed to compare the results at different levels of urodynamic testing in a cross section of spinal cord injured patients to determine what type of testing is required. A further goal was to attempt to elucidate differences among patients with areflexic detrusor function in order to predict which patients might void satisfactorily with strain/Credé assistance.

Thirty-three males and two females were included in the study. Basic urodynamic testing consisted of rapid-fill gas cystometry with simultaneous pelvic floor electromyography (EMG) and urethral pressure profilometry. A multi-function urodynamic examination was compared with the basic testing procedure. This consisted of medium-fill liquid cystometry using a radiopaque substance at room temperature. Intravesical and intraabdominal pressure were measured, as well as pelvic floor EMG activity and simultaneous fluoroscopic monitoring. It was determined that patients demonstrated to be areflexic on basic urodynamic testing did not have any significant additional information provided by the multi-function study. Furthermore, the urethral pressure profile was of no benefit in these patients and no predictive factors could be identified to determine which patients with areflexic bladders might void successfully by strain or Credé techniques. Patients with large volume reflex bladders most often had additional therapeutic information provided by the multi-function examination.

In general detrusor sphincter dyssynergia was shown to be more severe in the multifunction examination, and hence sphincterotomy was recommended. Bladder neck dyssynergia was also identified in three patients, and this could not be appreciated from the basic urodynamic testing. The current study did not enable us to determine any predictive factors which clearly indicated a need for surgical intervention. Since it is recognised that bladder function changes over time, the necessity for periodic reassessment is confirmed. 


\section{Management of the neuropathic bladder by chronic clean intermittent catheterisation -5 year outcomes \\ F. M. Maynard, M.D. and J. Glass, D.O. \\ University of Michigan Spinal Cord Injury Care System, Ann Arbor, Michigan, U.S.A.}

Between March and June 1985, 40 patients with a neuropathic bladder following traumatic spinal cord injury were contacted. They had all previously participated in a study of urologic outcomes following clean intermittent catherisation used during their initial medical rehabilitation period. Of 34 patients discharged using chronic clean intermittent catherisation, $28(82 \%)$ continued to use it. Three patients became catheter-free with or without an external collector, one following a sphincterotomy. One patient had regained normal voiding and two patients had electively changed to catheter drainage in order to improve their independence. There were no conduit procedures and no changes in urinary management due to upper tract complications in this group.

Based on the results of this study, the following conclusions were made:

1. Over $80 \%$ of patients using CCIC at discharge continued to use it, suggesting low morbidity and high patient acceptance.

2. Urological complications in patients using CCIC were more frequent in the lower urinary tract than in the upper urinary tract; were more frequent in men than women; and had a low morbidity.

3. Urological complications and hospitalisations were more frequent among the $41 \%$ of patients using CCIC who had frequent SxUTI's.

4. SxUTI's were common in patients using CCIC but rates may be comparable to other methods.

5. Although $44 \%$ of CCIC patients were hospitalised at least once in 5 years for urological complications, this is comparable to reported multi-center outcomes in the United States (Young, 1982).

Urological consequences of untreated bacteriuria in spinal cord injury patients K. V. Kuhlemeier, Ph.D., C. T. Huang, M.D., M. J. DeVivo, Dr P. H. and L. K. Lloyd Departments of Rehabilitation Medicine and Urology, University of Alabama at Birmingham, Birmingham, Alabama 35294, U.S.A.

The authors studied 337 spinal cord injured(SCI) patients at the Spain Rehabilitation Center, dividing them into four groups. Each group had two examinations, 1 to 2 years apart. Group A were those with indwelling catheters at the time of both examinations; Group B, those with sterile urine at the first examination; Group C, those with asymptomatic bacteriuria at the time of the first examination who were not treated with antibiotics; and Group D, those with asymptomatic bacteriuria at the first examinations who were treated with antibiotics according to the results of routine culture and sensitivity testing.

The results suggest that treatment of asymptomatic bacteriuria in spinal cord injured patients who may not be as advantageous to the patient as has been supposed. This is based on the fact that SCI patients with asymptomatic, but treated, bacteriuria did not fare appreciably better (in terms of incidence of urological complications and renal function) than similar patients whose asymptomatic bacteriuria goes untreated. Patients with untreated 
bacteriuria did have a higher incidence of therapeutic urological surgical procedures than patients with treated bacteriuria. However, the authors noted that this group had a longer mean duration of injury and therefore was more likely to develop the usual complications coincident with chronic spinal cord injury.

\section{Anticholinergic suppression of bladder reflex activity following complete suprasacral spinal cord injury \\ Rodney Anderson, M.D., Mary P. Hyland, Kenny Tikizawa, Conal Wilmot \\ Santa Clara Valley Medical Center, 3751 South Bascom Avenue, San fose, California 95128, U.S.A.}

The authors investigated 240 males and females injured from 1978 to 1982, seen in the Santa Clara Valley Medical Center and followed up in the outpatient urology service from 2 to 6 years. Patients were individually selected to receive anticholinergic therapy with or without accompanying alpha andrenergic blocking agents based upon monitor voiding cystourethrogram and voiding urodynamic studies. Patients using only intermittent catheterisation and anticholinergic suppression had an outpatient bacteriuria $\left(10^{4} / \mathrm{ml}\right)$ rate of 027 . Patients using condom drainage, with or without partial intermittent catheterisation, had an infection rate of 60 . There were significantly greater degrees of bladder deterioration in the form of trabeculation in those patients relying on reflex voiding than those using anticholinergic suppression and intermittent catheterisation.

Results indicated a trend towards a more normal urinary tract anatomy and less susceptability to urinary tract infection with the deliberate use of anticholingeric suppression of reflex bladder activity following spinal cord injury.

\section{Comparative study of electrophrenic nerve stimulation and mechanical ventilatory support}

R. Edward Carter, M.D.

Texas Institute of Rehabilitation and Research, American College of Medicine, Houston, Texas, U.S.A.

The author discussed 37 ventilatory dependent spinal cord injured persons who had been discharged from the Texas Institute of Rehabilitation and Research in the past 17 years. Nineteen of these were on mechanical ventilators and 18 on electrophrenic respirators. The following parameters were examined: mortality rates and causes, duration of follow up, and place of residence, as well as the usual demographics.

The author concluded that no single facility had a large enough number and experience with these types of patients to forecast appropriate respiratory maintenance and ultimate outcome. They suggested a centralised registry for all ventilatory-dependent spinal cord injured patients and were organising one at the Texas Institute of Rehabilitation and Research.

By this action, they hope to be able to answer questions concerning mortality, morbidity, reliability of equipment and equipment cost, place of residence, primary care givers in the home, and the potential for ultimate productivity. 


\title{
Total respiratory compliance: effect of body position in acutely injured quadriplegics
}

Chi-Tsou Huang, M.D., Keith V. Kuhlemeier, Ph.D., Michael F. DeVivo, Dr. PH., Samuel L. Stover, M.D., Philip R. Fine, Ph.D.

Spain Rehabilitation Center, University of Alabama at Birmingham, Birmingham, Alabama 35294, U.S.A.

The objective of this study was to determine the effect of body position on total respiratory compliance and inspiratory volume and to assess the impact of IPPB on the end-tidal $\mathrm{CO} 2$ fraction in acute spinal cord injury patients in the supine, sitting and $20^{\circ}$ head-down positions. Total respiratory compliance consists of lung, thoracic cage and diaphragmatic compliances.

Eleven quadriplegic patients and 12 controlled subjects comprised the study population. The authors concluded that IPPB therapy with a flow rate of five litres per minute and pressures up to $20 \mathrm{~cm}$ of water provides an appropriate means of inducing alveolar $\mathrm{CO} 2$ retention and makes use of maximal respiratory compliance in acutely injured quadriplegic patients. Therapeutic efficacy of IPPB is largely unaffected by body positions.

\author{
Home care of ventilatory dependent children-the University of Michigan \\ experience \\ Virginia S. Nelson, M.D. \\ University of Michigan Hospital, 1 D204 University Hospital, 1500 East Medical Center Drive, \\ Ann Arbor, Michigan 48109, U.S.A.
}

Twelve physically handicapped ventilatory dependent children (responauts) aged 7 months to 17 years were discharged with full respiratory support from the University of Michigan Mott Children's Hospital. Respiratory failure was caused by spinal cord injuries ( 9 quadriplegics, one paraplegic patient with phrenic nerve rupture), spinal muscular atrophy, and Zellweger's syndrome. Ten responauts were discharged with positive pressure ventilators, one with a cuirass, one with both an iron lung and a cuirass. At home responauts required 24 hours of care daily from parents or home care providers with nursing care from agency personnel ranging from 0 to 24 hours per day. Responauts were ventilator dependent 11 to 24 hours per day at the time of discharge.

Review revealed that in the first $4 \frac{1}{2}$ years after discharging responauts home, there have been 46 readmissions of those who were at home for at least six months, with an average of 4.2 per child or 1.9 per child year at home. These readmissions have accounted for 534 days of hospitalisation. Reasons for readmission have been surgery (12), respiratory problems including pneumonia (8), urosepsis (4), other infections (6), seizures (1) and miscellaneous (6). Numerous problems were encountered in discharging responauts including funding, equipment, psychosocial, and attendant availability, but these have been solved through the co-operation of families with the hospital rehabilitation team, home care agencies, funding agencies and schools. Benefits derived from home care include social interaction, developmental growth, and strengthening of family bonds. 
Glottic and tracheal stenosis in spinal cord injured patients

Shih-Fong Hsu, M.D., James N. Dreisbach, M.D., Susan W. Charlifue, M.A., Gerald M. English, M.D., F.A.C.S.

Craig Hospital, 3425 South Clarkson, Englewood, Colorado 80110, U.S.A.

Patients with spinal cord injury may require tracheostomy and/or intubation for: (1) protection of the airway; (2) maintenance of the airway; or (3) pulmonary ventilation and hygiene. The authors studied retrospectively 56 patients with those problems.

Diagnosis of glottic and subglottic tracheal stenosis was made by clinical examinations, air contrast laryngograms, polytomography, CT scans, and endoscopic examinations. Patients were classified into four groups. Those with less than $20 \%$ stenosis were placed in Class I, 20 to $50 \%$ stenosis were placed in Class II, 50 to $80 \%$ in Class III and those with more than $80 \%$ in Class IV. Twenty-four Class I, 13 Class II, 9 Class III, and 10 Class IV. Treatment consisted of endoscopy, excision of granulation or cicatrical tissue, dilation of the glottis and/or trachea, tracheotomy, tracheal T-tubes, tracheoplasty and laryngeal keels.

Results of therapy appeared to depend on several factors including the severity of the stenosis and associated illness. Sixteen patients in Class I were eventually successfully extubated and eight in whom a tracheotomy tube was necessary. Among Class II patients, six were extubated and seven required tracheotomy. In these two groups of patients, the level of the injury and associated pulmonary problems were the primary reasons prohibiting extubation. There were seven Class III patients who were extubated and two who required tracheotomy. These two patients required a tracheotomy for inadequate tracheal airway. Only three Class IV patients have been extubated and the other seven required tracheotomy.

\section{Vibratory stimulation of the penis in men with spinal cord injury George Szasz, M.D. \\ Sexual Medicine Unit, Faculty of Medicine, University of British Columbia, Vancouver, British Columbia, Canada}

Seventeen men with lesions above $\mathrm{T} 12-\mathrm{Ll}$ vertebral levels responded to vibration with some degree of predictable series of pelvic sensory experiences, abdominal leg muscle spasm, elevations of blood pressure and increase in the pulse rate. Fourteen of these men ejaculated seminal fluid containing various quantity and quality of spermatozoa. Three of five persons with lesions above $\mathrm{T} 4$ ejaculated but experienced a mild degree of autonomic hyper-reflexia. Five men with lesions below T12-Ll vertebral level did not show a response to vibration. The authors concluded that vibratory stimulation of the penis in men with spinal cord injury with intact sacral lumbar low thoracic segments may produce a predictable series of sensory and neuromuscular reaction and may lead to ejaculation of seminal fluid. This suggested that part of the neurological mechanism for ejaculation is located in these segments. Autonomic dysreflexia is a potential danger in patients with high spinal cord lesions. The vibration can create responses with may be interpreted by some men with spinal cord injury as sexual. Seminal fluid obtained with this method has a potential for use in artificial insemination. 
Sexuality and disability clinic: preliminary experience with electroejaculations in spinal cord injured man

Lauro S. Halstead, M.D., Stephen W. J. Seager, M.A., Shane VerVoort, M.D. 1333 Moursund Avenue, Texas, Houston, Texas 77030, U.S.A.

Eight patients, five paraplegics and three quadriplegics with an age range of 23 to 38 years and 6 to 13 years post onset of injury were evaluated following the use of rectal probe electroejaculation. Electroejaculation was performed in the outpatient clinic using $11 / 8$ to $1 \frac{1}{4}$ inch rectal problems precision machine with solid bars of PVC. Antegrade ejaculation occurred in seven subjects (four paraplegics and three quadriplegics) with impairment proven sperm count and morphology on repeated stimulations. No significant retrograde ejaculation occurred in any of the subjects. Sperm acceptable for artificial insemination was obtained from one subject. The major side effects were mild dysreflexia in one subject and disruption of normal bowel program in one subject. The authors concluded that electroejaculation is a safe, relatively brief outpatient procedure and with repeated stimulation has a good potential for producing sperm acceptable for artificial insemination in selected patients.

Effects of spinal cord stimulation on spinal spasticity

Giancarlo Barolat, M.D. and Joel M. Myklebust, Ph.D.

Department of Neurosurgery, Thomas fefferson University, 1015 Walnut Street, Philadelphia, Pennsylvania 19107, U.S.A.

Sixteen patients with severe spasms secondary to traumatic myelopathy underwent epidural Spinal Cord Stimulation. The patients had previously undergone extensive trials with medications and physical therapy with unsatisfactory results. In two subjects, satisfactory electrode placement could not be obtained and there were no positive therapeutic results. All 14 of the other patients had at least a $50 \%$ reduction in the severity of their spasms and, in 8 patients, spasm was reduced by more than $75 \%$. In 8 patients, the reduction or abolition of spasms occurred within seconds or minutes from the onset of stimulation, and the effect disappeared within minutes after cessation of stimulation. In the other 6 patients, the effects became evident only after several hours of stimulation. The spasms were affected in the upper and lower extremities as well as in the truncal and the abdominal muscles. Marked improvement in bladder and bowel function was observed in two patients. There was no neurological deterioration following the operative procedure or the long term use of spinal cord stimulation.

Complications included infection in one patient, two electrode migrations, two wire breakages, and one skin breakdown at the connector site. All the complications and technical problems were corrected through simple procedures under local anaesthesia. The authors conclude that spinal cord stimulation is an effective method of treating spasms due to myelopathy, its greatest value lies in the management of patients with incomplete myelopathy and good functional status. 
Further experience with nifedipine in the treatment of autonomic dyseflexia Rosemary Lindan, M.D., Elizabeth Leffler, R.N., Alvin Freehafer, M.D., Anne Marie Lyons, R.N. and Coletta Hazel, R.N.

Spinal Cord Injury Service, Cuyahoga County Hospital, Highland View, Cleveland, Ohio 44109, U.S.A.

The authors evaluated 22 of 26 patients treated with nifedipine for autonomic dysreflexia (A.D.) over a 2-year period. All the patients were quadriplegic from $\mathrm{C} 3$ to $\mathrm{C} 7$. The standard procedure observed for patients exhibiting A.D. was the following:

1. Raise the head of the bed, or sit up to $90^{\circ}$ angle.

2. Monitor blood pressure while identifying the cause of autonomic dysreflexia. Continue to monitor until stable. Record on the appropriate form.

3. Administer $10 \mathrm{mg}$ nifedipine orally or sublingually.

4. Check bladder/bowel and empty as needed.

5. If the blood pressure is still elevated after 30 minutes, give another $10 \mathrm{mg}$ nifedipine.

6. Check for other causes.

7. Continue to observe the patient closely for signs and symptoms of autonomic dysreflexia for 8 hours.

Using this procedure, it was not necessary to use any other parenteral hypotensive medication to control autonomic dysreflexia. In most instances the blood pressure returned to safe levels within 10 to 15 minutes and to normal levels within 30 minutes regardless of whether or not an identifiable cause had been found and relieved.

The authors concluded that nifedipine given orally or sublingually in doses of 10 to $20 \mathrm{mg}$ is a safe and effective agent for the treatment of autonomic dysreflexia.

\author{
Decreased theophylline bio - availability and impaired gastric emptying in \\ spinal cord injury \\ Jack L. Segan, M.D., Norah Milne, M.D., Sherry R. Brunnemann, B.S., Stanley K. \\ Gordon, M.D., Ibrahim M. Eltorai, M.D. \\ Veterans Administration Medical Center (111GM), 5901 East Seventh Street, Long Beach, \\ California 90822, U.S.A.
}

This study was implemented in order to evaluate the bio-availability of orally administered theophylline in a population of humans characterised by disorders of gastrointestinal motility. Thirty-eight male volunteers within $10 \%$ of ideal body weight with normal cardiac, renal and hepatic function as confirmed by physical examination, blood screening and urinalysis were studied. Eleven were paraplegic and 17 were tetraplegic. Ten subjects with intact neuraxes served as able bodied controls. All spinal cord injuries were traumatic, greater than 1 year duration, and physiologically complete as defined by history of dysreflexia, absence of voluntary motor function, loss of sensation and environmentally integrated sweating below the level of the lesion. The authors demonstrated that the absolute bioavailability of oral theophylline in spinal cord injury appears to be decreased when an impairment in gastric 
emptying of liquid meals exists. The magnitude of the change varies with the level of cord injury and is greatest in those with cervical myelopathies.

These findings were supportive of a casual relationship between the impairment of gastric emptying which had been observed in tetraphlegia and the decrease in bio-availability and rate of absorption of theophylline which was demonstrated in this study. The results suggest that altered theophylline bio-availability and chronic impairment of gastric emptying are characteristic of spinal cord dysfunction and is bimodally distributed in those with a high myelopathy.

Individualised digital bowel assessment of the spinal cord injured patient Adrienne E. Johnson, R.N., Mattie Ogburn and Yvonne Flowers, R.N. Rehabilitation Institute, 261 Mack Boulevard, Detroit, Michigan 48201, U.S.A.

The digital bowel assessment protocol involves the exploration of five parameters:

1. Anal sphincter tonne.

2. Status of the anal vault.

3. Internal sphincter tone.

4. Status of the lower rectum.

5. Positional relationship of the internal and external sphincters.

For this plan, the authors used no enemas and few suppositories in duration evacuation with 10 to 20 minutes rather than an hour or more used for traditional approaches. Fifty patients who received assessment management according to the protocol were compared to 39 patients who had traditional bowel management. In the latter group, $7(18 \%)$ had one or more episodes of hyperreflexia, $4(10 \%)$ had severe impaction, $4(10 \%)$ had chronic constipation, and $10(26 \%)$ had frequent incontinence. None of these complications were observed for the first group. The authors concluded that a systematic approach to bowel assessment and management improved the ability of patients to relieve an effective bowel program.

Identification of herniated nucleus pulposis in spinal cord injury patients

David F. Apple, Jr., M.D., Allen P. McDonald, M.D., Richard A. Smith, M.D.

Shepherd Spinal Center, 2020 Peachtree Road, N.W., Atlanta, Georgia 30309, U.S.A.

In 10 years, 657 patients with cervical spinal cord injury were treated at the Shepherd Spinal Center. During this time, five cases of symptomatic herniated disk following injury were documented and reviewed. This provided an incidence of $7 \%$ of cervical injuries and $4 \%$ of all injuries. Common characteristics were radicular pain in four, incomplete cord syndromes in four, diagnosis by positive myelogram in four, and bilateral facet dislocations in three with a $2.3 \%$ incidence. 
Paraplegic patients achieve stair climbing and reciprocal walking by means of functional electrical stimulation (FES) and crutches

Professor Alojz Kralj, Professor Tadej Bajd, R. Turk and Helika Benko, Janez Sega

Pritzker Institute of Medical Engineering, Illinois Institute of Technology, Chicago, Illinois, U.S.A.

In this study, only four channels of stimulation were employed. Two for bilateral quadriceps and two for sensory stimulation for flexion reflex triggering. Quadriceps stimulation is used for knee locking and providing anti-gravity support. The flexion reflex withdrawal movement provides the swing phase of gait. The investigators did not adopt the usual mode of preprogramed FES sequences or the closed loop approach, but chose the principle that the patient is controlling the gait events and closing the loop. From the results, the authors concluded:

1. The adopted patient closed loop principle is to be favoured because of the advantages it presents.

2. The flexion reflex may also provide adequate swing phase movements for stair ascension.

3. The minimum required number of FES channels for reciprocal stair ascension equals the adopted four channel level walking set up.

Variations in the return of motor control following functional electrical stimulation in spinal cord injured patients

Anne L. McDermott, L.P.T., Gilbert Brenes, M.D., John Sikora

Harmarville Rehabilitation Center, P.O. Box 11460, Guys Run Road, Pittsburg, Pennsylvania 15238, U.S.A.

This investigation was undertaken to evaluate the efficacy of strengthening voluntary muscle movements after spinal cord injury and to identify optimal treatment regimes. Patients with neurologically complete injuries received stimulation to muscle groups one level below their lesion in the hope of facilitating motor return due to the phenomenon of nerve root escape. Neurologically incomplete injuries received FES to any muscle group having a reasonable potential for return. Findings indicate a positive significant correlation between the total number of sessions and the increase of strength as measured by manual muscle tests. There were significantly greater strength gains for incomplete compared to those with complete injuries.

Analysis of variance was performed to compare the stimulated cases with the controls by completeness of injury for six functional categories including wheelchair activities, wheelchair propulsion, transfers, mobility, balance and ambulation. The stimulated incomplete cases had significantly greater degrees of improvement for wheelchair propulsion, mobility, transfers and ambulation than did the control group. Patients with complete injuries only demonstrated a significantly higher improvement in the area of wheelchair propulsion when compared with the non stimulated controls. 


\section{A collaborative study of high quadriplegia}

Gale G. Whiteneck, Ph.D., R. Edward Carter, M.D., Susan W. Charlifue, M.A., Karyl

M. Hall, Ed.D., Robert R. Menter, M.D., Margaret Ann Wilkerson, B.S., Conal B.

Wilmot, M.D.

Craig Hospital, 3425 South Clarkson Street, Englewood, Colorado 80110, U.S.A.

An in-depth follow up investigation of high quadriplegics was conducted by three collaborating regional spinal cord injury systems: The Northern California Regional Spinal Cord Injury System, The Rocky Mountain Regional Spinal Cord Injury System, and the Texas Regional Spinal Cord Injury System.

The population studied consisted of $216 \mathrm{C} 4$ and higher level quadriplegic individuals with complete injuries occurring between 1973 and 1983. During the initial SCI System hospitalisation, $62 \%$ used mechanical ventilator assistance but by discharge, only $35 \%$ required only full or part-time ventilator assistance. The remaining $55 \%$ were ventilator independent at discharge. Seventeen patients $(8 \%)$ expired during the initial hospitalisation. The survival rate was investigated at different intervals with $73 \%$ surviving at 9 years post injury. Total days in both system and non-system hospitals for the entire study population averaged 13.4 days per person per year. The respirator dependent individual had an average of 22.2 days whereas the respirator independent group averaged 10.5 days.

Regarding activities, the respirator dependent group appeared as active as the respirator independent group although they got out of the house less often. Drug and alcohol abuse were low and suicide was not a prevailing theme in their lives. With regards to cost, the initial SCI system rehabilitation expenses averaged $\$ 181,929.00$ for respirator dependent cases, and $\$ 64,553.00$ for respirator independent cases. Adding the cost of system and non-system in patient out patient and physician charges, equipment charges, modification expenses, medication and supply charges, and other miscellaneous expenses brought the grand total for annual follow up care to a mean of $\$ 101,246.00$ for respiratory dependent cases, and $\$ 27,607.00$ for the respirator independent cases. From the study, it was clear that high quadriplegics are a substantial segment of the SCI population and that with proper care they are surviving and living satisfying lives.

\section{Dynamic effects of Functional Electrical Stimulation (FES) for the prevention of pressure sores}

Simon P. Levine, Ph.D., Frederick M. Maynard, M.D., Lisa D. Bowers, M.S., Ronald L. Kett, M.S.

University of Michigan Medical Center, Ann Arbor, Michigan 48109-0032, U.S.A.

The authors designed these pilot studies to study the interface pressure variation and tissue undulation produced as immediate dynamic effects of FES. Various pressure measurements were made using two different pressure measurement pads. Tissue shape changes were measured during ultrasonic imaging. These pilot studies on five able-bodied subjects demonstrated that FES can vary interface pressures, produce tissue undulation and reconfigure the shape of the buttocks under load. The pressure measurements, which have a great deal of inherent error, provided a semi-quantitative indication of changes in tissue configuration. The ultrasound experiments provided a more direct measure of the tissue configuration changes. This tissue undulation was hypothesised to be able to dynamically allow blood flow to ischemic areas and to help prevent pressure sores. The authors felt that similar results would be expected in paralysed individuals with only slight to moderate muscle atrophy. They felt 
that those with appreciable muscle atrophy would require chronic stimulation for reconditioning before similar results could be expected.

\section{Pressure sore readmission program: a method to reduce chronic readmissions for pressure sore problems}

Janet G. LaMantia, M.A., R.N., C.R.R.N., Judith F. Hirschwald, M.S.W., Carol L. Goodman, B.S.N., C.R.R.N., Vernice Wooden, M.S.S.A., William E. Staas, Jr., M.D. Magee Rehabilitation Hospital, Six Franklin Plaza, Philadelphia, Pennsylvania 19102, U.S.A.

A pressure sore readmission program was designed to heal existing severe, and/or chronic skin problems to prevent further occurrence. The program was created to respond to the Magee Center's experience of an $80 \%$ recurrence of pressure sores within 3 months of successful treatment. Prospective patients participated in a comprehensive medical, nursing and psychosocial assessment to determine medical status and patient goals and motivation. The program was designed in three phases: pre-surgical, surgical and post-surgical.

1. Presurgical: lasting 7 to 10 days where emphasis was placed on preparation for surgery, assessment of functional ability and identification of individual patient goals.

2. Surgical: lasting 10 to 14 days and involving acute care hospital admission.

3. Post-surgical: where the patient was required to attend classes, therapy and other activities such as a coping group and a patient team meeting on a weekly basis.

Points were earned by patients through participation in classes, therapies and defined aspects of personal care. A failure to earn point quotas or an inability to comply with the hospital or program policies resulted in a contract violation with four violations resulting in premature discharge. Following discharge, weekly monitoring occurred for the first month. If the skin remained intact, the patient was then monitored monthly until 3 months post discharge and then annually on a routine follow up schedule.

Results indicated 36 patient admission to the program, $75 \%$ being paraplegic, $64 \%$ of these being complete. Twenty-three patients completed the program with 17 remaining healed at 3 months. Three patients had reopened skin and 3 patients were not evaluated. The MannWhitney $U$ distribution was employed to determine associations between program components and healed skin. There was a positive association between high test scores and healed skin at 3 months. Positive association was also found between compliance with behavioural expectations and healed skin at 3 months. There was no significance between healed skin at 3 months and the parameters of psychosocial intervention-attendance at psychosocial intervention sessions and the quality of participation by a patient in individual and group sessions. Healed skin at 1 year did not have a positive association with significance for any of the program parameters.

\section{Outreach clinic program: method and outcome analysis in spinal cord injured persons}

Kimberley Shanahan, R.N., Jennie Evans, P.T., Cindy Burns, M.Ed., Lesley M. Hudson, M.A., David F. Apple, Jr., M.D.

Shepherd Spinal Center, 2020 Peachtree Road, N.W., Atlanta, Georgia 30309, U.S.A.

Outreach program was implemented in 1975 in which a comprehensive rehabilitation team including a physician, nurse, physical therapist, counsellor, recreation therapist, and 
co-ordinator, was sent into the field with the objective of identifying, evaluating and treating those patients who had been lost to follow up by the Outpatient Services Department. A demographic analysis of the catchment area yielded clusters of patients lost to follow up in key geographical areas.

Appropriate hospitals within those areas were selected to co-operate in an Outreach Clinic Program. Eighty-three patients were evaluated in 13 different clinics. Medical problems identified were contractures in $66 \%$, decubiti in $35 \%$, obesity $27 \%$, pain $54 \%$, spasticity $45 \%$, and urinary tract infections in $40 \%$. The prominent problem identified by the rehabilitation counsellor was unemployment in $79 \%$. The patient evaluator revealed that $96 \%$ felt that the Outreach Clinic Program was helpful because it was more convenient to patient location and it brought a medical team familiar with the special needs of the spinal cord injured population to the patient's home area.

\author{
Evaluation of patient learning in a spinal cord education program \\ Roberta J. Phillips, R.N., C.R.R.N. \\ Santa Clara Medical Center, 751 South Bascom Avenue, San fose, California 95128, U.S.A. \\ Karyl M. Hall, Ed.D. \\ Veterans Administration Medical Center, Palo Alto, California, U.S.A.
}

This study was designed to determine whether a systematic education program had a significant effect on patient learning and whether this information was retained several months later.

Eighty patients in the spinal cord injury program at Santa Clara Valley Medical Center were given a self care test before and after a comprehensive series of classes was offered over several weeks' time. Fifty-five of those evaluated initially were evaluated at 1 year post discharge. These results were compared with pre and post education course scores. Results shows a highly significant difference between pre and post test scores and between pre and follow up test scores. Class attendance was correlated with post-test performance and with educational level. Class attendance was significantly related to post test performance. The more classes a patient attended, the better he or she did on the post test. Education level of patients was significantly related to pre and post test and follow up scores although more strongly to pretest scores. The higher the education before injury, the better performance on the test.

However, educational level also was significantly related to attendance. It was felt that attendance was the major factor in test performance rather than educational level. Poorpremorbid academic history (i.e., defined as people who dropped out of school, failed, or were diagnosed as learning disabled) was not a factor in pretest scores or pre to post test change scores. There was found to be a relationship between neuropsychological scores at both pre and post education scores so that the more impaired they were shown to be on neuropsychological scores, the more poorly they performed on the patient education pretest and follow up test. It was suspected that a mild head injury sustained at the time of the accident may play a role in how well patients perform. It is important to note that the amount the impaired patients learned (as reflected in the change score between pre and post tests) was not significantly different from those without cognitive deficits.

The authors concluded that classes do provide a more thorough education than ward exposure, and patients learn and retain vital self care information in pointing for the need for a comprehensive structured learning program in the rehabilitation setting. 


\section{Spinal cord injury unit nurses teach patient and families}

Yvonne Flowers, R.N., Adrienne Johnson, R.N., Frances Davis, R.N., B.S.N.

Rehabilitation Institute, 261 Mack Boulevard, Detroit, Michigan 48201, U.S.A.

This study reports on a 2 year experience of nurses in a specialised spinal cord injury rehabilitation unit regarding information on family members involved, subjects taught, and the outcome of teaching in seven areas. The study was undertaken on 151 patients. Subject areas were catheterisation, skin checks, signs and symptoms, nutrition, transfers, hygiene, and bowel care. The results indicated that interest and understanding were highly correlated. Family interest is predictive of patient interest, and family understanding is predictive of patient understanding.

\section{A profile of denials of durable medical equipment for spinal cord injury patients by third party payers}

William H. Donovan, M.D. and R. Edward Carter, M.D.

The Institute for Rehabilitation and Research, 1333 Moursund Avenue, Houston, Texas 77030, U.S.A.

A letter was sent to 259 members of the American Spinal Injury Association asking them to list representative cases where requests for equipment deemed necessary were denied. Twenty-eight responses from different rehabilitation facilities were received from 16 States describing 110 patients and 176 pieces of durable medical equipment for which payment was denied in 1984 and 1985.

The two most common reasons for denial were: it was not covered by the insurance policy $(36 \%)$, and it was not medically necessary $(31 \%)$. Ninety pieces of equipment $(51 \%)$ were eventually obtained, most often from a charity or through the patient's family resources. For three pieces, a lawyer's assistance was obtained. The items most frequently denied were the most fundamental for mobility for a paralysed person-wheelchairs.

\section{Do rehabilitation staff misperceive depression in spinal cord injury patients? Marcel Dijkers, Ph.D., Cynthia Harrison, B.S., Laura Cushman, Ph.D. Rehabilitation Institute, Department of Research, 261 Mack Boulevard, Detroit, Michigan 48201, U.S.A.}

The aim of this study was to investigate accuracy of perceptions and explore the nature of and variations in staff perceptions of depression in SCI patients. A questionnaire was completed by 71 rehabilitation staff members from various disciplines with an average of 6 years experience in their profession, 4 years in rehabilitation and 3 years in spinal cord injury care. The conclusion was that there was no unitary view of depression in SCI patients among rehabilitation staff. The staff varied in their reporting of the symptoms of depression, the importance they lend to these symptoms and the intra and inter-individual variation in depression they perceive.

Finally, the staff perceived depression in SCI patients with some accuracy but tended to overestimate it. 
Automatic negative thoughts associated with depression in traumatically injured spinal cord patients

Craig J. Alexander, PhD.

Kessler Institute for Rehabilitation, Inc., 1199 Pleasant Valley Way, West Orange, New fersey 07052, U.S.A.

Beck's cognitive theory of depression states that people are thinking beings that interpret events in life. These interpretations are based largely on the individuals schemata or characteristic ways of organising, construing or interpreting events. According to this model, the spinal cord injured patient might engage in various maladaptive cognitive styles along with irrational/dysfunctional self-statements which contribute to the etiology and maintenance of depression. Cognitive psychotherapy attempts to systematically identify. and alter negative automatic thoughts.

The author's study was designed to assess maladaptive cognitions in 20 traumatically injured male spinal cord patients ranging in age from 18 to 32 . Patients were classified as depressed or non-depressed on the basis of their Beck Depression Inventory scores taken 3 to 5 months post-injury. The Automatic Thoughts Questionnaire was administered to all patients to assess for maladaptive cognitions. Depressed patients scored significantly greater on 8 of the 30 maladaptive self-statements. Although these 8 items are dysfunctional they seem quite rational for spinal cord injured patients. The author concluded that the usual cognitive criteria used for diagnosing depression may not hold true in a rehabilitation setting. That is, what is considered maladaptive and irrational cognitions for psychiatric patients may be quite appropriate for spinal cord injured patients. The results pointed out the need for rehabilitation specific assessment devices, especially for depression.

The determinants of blood pressure in a spinal cord injured population Stephen H. Dearwater, M.S., Lucille S. Adams, Ph.D., Gilbert Brenes, M.D., Ronald E. LaPorte, Ph.D., Daniel D. Savage, M.D., Ph.D., Richard A. Washburn, Ph.D. University of Pittsburgh, Pittsburgh, Pennsylvania, U.S.A.

The authors examined the factors associated with blood pressure in the spinal cord injured person. Twenty-four paraplegics and 28 quadriplegics, ages 29 and 28 respectively were studied. Twenty-nine per cent of the paraplegics and $10 \%$ of the quadriplegics had a diastolic blood pressure greater than $90 \mathrm{mmHg}$ whereas $12 \%$ and $7 \%$ of the paraplegics and quadriplegics respectively had systolic blood pressures greater than $140 \mathrm{mmHg}$. The systolic and diastolic blood pressures were significantly higher in the paraplegics than the quadriplegics. The mean physical activity level as expected was higher in the paraplegics. The leaner, more active paraplegics demonstrated higher resting systolic blood pressures than the more sendentary paraplegics. There was no predictor of systolic blood pressure or diastolic blood pressure for the quadriplegic. The risk factors commonly linked with able bodied hypertension: age, obesity, smoking, alcohol consumption, were not factors in either SCI group. 
Alcohol use and activity patterns following spinal cord injury

Allen W. Heinemann, Ph.D., Mary Keen, M.D., Sidney Schnoll, M.D., Ph.D., William Adair, M.D.

Rehabilitation Institute of Chicago, 345 East Superior Street, Chicago, Illinois 60611, U.S.A.

Use of, dependence on, and addiction to alcohol are characteristics that influence life adaptation of persons without physical disabilities. In persons who acquire a physical disability, adaptive deficits, reflected in the use of alcohol before disability, may predict a poor rehabilitation outcome during and following inpatient rehabilitation care.

A group of 30 patients was analysed. Alcohol consumption was quantified by asking participants to report frequency and quantity of alcohol use on weekdays and weekends during the 6 months prior to injury. Activity pattern information was collected over a weekday and weekend day for each participant. The activity with the longest duration was sleeping followed by personal care, television viewing, rehabilitation, socialising, other quiet recreation, and other quiet activities such as smoking or drinking. People who drank during the 6 months prior to injury watched television more frequently, travelled less frequently, spent less time sleeping and spent more time engaged in quiet recreational activities during their hospitalisation than did persons who drank less before their injuries. No differences were found in the frequency or duration of time spent in rehabilitation related activities.

The authors concluded that persons with histories of greater alcohol use spend their time differently during inpatient rehabilitation than do patients with less alcohol use, though time spent in rehabilitation performing activities and activities without supervisory assistance was unrelated to alcohol use.

Treatment of femoral and tibial shaft fractures occurring in patients with acute spinal cord injuries

Douglas E. Garland, M.D.

Chief Spinal Cord Injury Service, Rancho Los Amigos Medical Center, 7601 East Imperial

Highway, Downey, California 90242, U.S.A.

Twenty-seven femoral shaft fractures in 23 patients and 34 tibial fractures in 28 acute spinal cord injured patients were evaluated to determine the outcome of operative versus nonoperative treatment. Analysis of the results of fracture care and medical complications such as pressure sores contributed to the recommendation that fractures of long bones in the lower extremity be considered for open reduction and internal fixation regardless of the neurological diagnosis when the patient is medically stable. Contrary to previous reports, these fractures did not routinely heal rapidly with non-operative treatment and in fact prolonged healing times were noted, with a propensity towards delayed and non-union. Open reduction and internal fixation appears to enhance healing, allow the most predictable outcome concerning fracture alignment, limb length and joint motion, whilst simplifying overall medical and nursing care and rehabilitation. 